\title{
Supersonic COIL with Nozzle Bank Based on Trip-Jet Mixing System
}

\author{
Kazuyoku TEI, ${ }^{1}$ Kazuhiko HORIOKA, ${ }^{2}$ Shigeki MUTO, ${ }^{1}$ Daichi SUGIMOTO, ${ }^{1}$ Goro WATANABE, ${ }^{1}$ and \\ Tomoo FUJIOKA ${ }^{1}$ \\ ${ }^{1}$ Tokai University, 1117 Kitakaname, Hiratsuka, Kanagawa 259-1292 \\ ${ }^{2}$ Tokyo Institute of Technologyncy, 4529 Nagatsuda-cho, Midori-ku, Yokohama 226-8503
}

(Received February 13, 2008)

\begin{abstract}
Nozzle banks for an ejector-chemical oxygen-iodine laser consisting of two-dimensional slit nozzles with a trip-jet mixing system were tested in the cold- and hot-flow operation regimes. Horizontal Pitot scan experiments demonstrated that the mixing ability of the trips is excellent. The Mach number of the mixed flow was approximately 3 . The gain measurements were conducted, and the results of measurements revealed that the maximum gain was around $0.6 \% / \mathrm{cm}$. The gain cut-off length was more than $200 \mathrm{~mm}$. The lasing experiments were also conducted, and the power was about $3 \mathrm{~kW}$ at the chemical efficiency of about $20 \%$.
\end{abstract}

Key Words: Ejector, COIL, Trip, Oxygen iodine laser, Pressure recovery

\section{Introduction}

One of the most important issues in chemical oxygen-iodine laser (COIL) development is the downsizing of exhaust system. An ejector COIL scheme, which uses high stagnation pressure $\mathrm{N}_{2}$, allows to increase recovery pressure up to approximately 100 Torr. Different types of ejector nozzles have been proposed. Nikolaev et al. used under-expansion high-pressure nitrogen flow to produce laser gas. ${ }^{1,2}$ A relatively large nitrogen flow (7-11 times that of oxygen) is necessary in order to achieve 80-100 Torr of cavity Pitot pressure, because the design point of the nitrogen stagnation pressure is lower (approximately 2-5 atms) than a nitrogen condensation limit. Yang et al. used a strategy to increase the nitrogen stagnation pressure, whereby their nozzle bank is based on two-dimensional slit nozzles, which can minimize total pressure loss, with mixing enhancement tabs mounted at the nozzle exit plane (NEP). ${ }^{3)}$ The stagnation pressure for nitrogen is approximately 7 atms, which enables a cavity Pitot pressure of 100 Torr at an oxygen dilution ratio of only 5 . Since iodine is premixed with the high-pressure nitrogen flow, however, their nozzle concept requires a high-pressure iodine supply system. ${ }^{4)}$ In addition, rapid expansion of the high-pressure $I_{2} / N_{2}$ flow caused iodine condensation. They recently proposed an iodine cluster buster simply with wires. ${ }^{5)}$

The nozzle bank reported herein is based on the two-dimensional slit nozzle array with mixing acceleration achieved by trip-jets (Fig.1). The mixing enhancement technique based on the trip-jets is originally used in HF/DF lasers. ${ }^{6,7)}$ In the case of COIL, iodine is supplied through the trip-jet injectors in order to prevent iodine condensation and to reduce the pressure of $\mathrm{I}_{2}$ supply line. The injection scheme we employed here is what we call uni-lateral injection, where the trip-jets are not injected toward $\mathrm{O}_{2}$ flow but only toward the high pressure $\mathrm{N}_{2}$ flow. This is because the nitrogen flow practically has the momentum to produce streamwise vorticity but not the oxygen flow. In addition, the trip injection into the $\mathrm{O}_{2}$ flow can increase the stagnation pressure for the oxygen flow, resulting in declined $\mathrm{O}_{2}\left({ }^{1} \Delta\right)$ yield. The nozzle banks for an ejector-chemical oxygen-iodine laser consisting of two-dimensional slit nozzles with a trip-jet mixing system were tested in the cold- and hot-flow operation regimes

\section{Cold-flow tests}

A cold-flow horizontal Pitot scan was conducted in order to evaluate the mixing ability of the trip-jets. For simplicity with regard to the test facility, nitrogen, rather than oxygen, was used for the primary flow. For the test a three-blade nozzle bank with $5.0 \mathrm{~mm}$ jet-to-jet distance was fabricated. Three Pitot probes covering different heights are integrated into one unit. The results for a 5\% trip flow rate are shown in Fig. 2 where the Pitot profiles measured at different scanning positions from the NEP are overlapped in the figure. The peak/valley structure almost disappears at $243 \mathrm{~mm}$, indicating that the mixing is complete. At this point, the Pitot pressure was more than 120 Torr, while the total pressure was approximately 300 Torr. In order to perform direct comparison, a nozzle bank with mixing tabs was also fabricated and tested

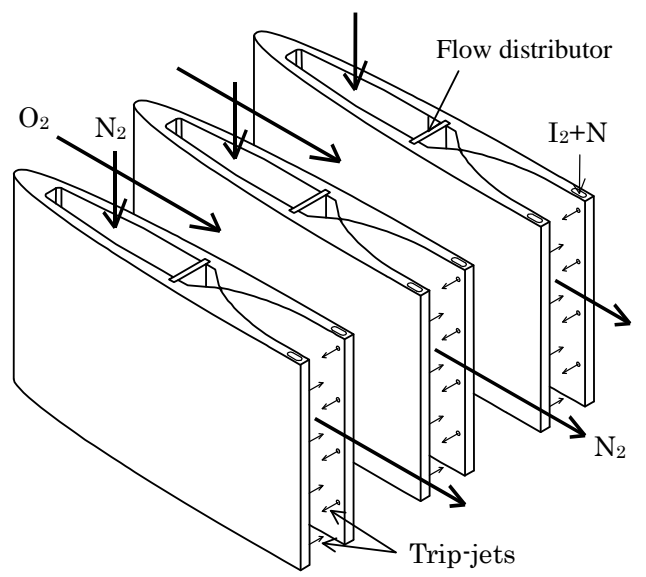

Fig. 1 Schematic drawing of the trip-jet ejector nozzle bank. 
in a cold run.

Maximum and minimum Mach numbers estimated from the Pitot scans are shown in Fig. 3 as a function of distance from the NEP. The mixing enhancement provided by trip-jets is comparable to that provided by mixing tabs. At the distance of $220 \mathrm{~mm}$ from the NEP, the Mach number of mixed flow for the trips and the tabs are 3.0 and 3.5, respectively. In addition, the results of the CFD analysis are shown in the figure. As expected, the mixing speeds obtained in the experiments are somewhat higher than those obtained by CFD, since the CFD calculation is based on laminar model.

In addition to the mixing evaluation for trips, backpressure tests were conducted in order to estimate the pressure recovery capacity. The cavity wall pressure was measured at a distance of $43 \mathrm{~mm}$ from the NEP The cavity pressure remained constant at approximately 10 Torr until the supersonic diffuser break back, and cavity unstart occurred at a backpressure of approximately 80 Torr. Test results for different trip flow conditions (including those for the tab nozzles) are summarized in Table I. It is interesting to note that the difference between the start pressure and the unstart pressure

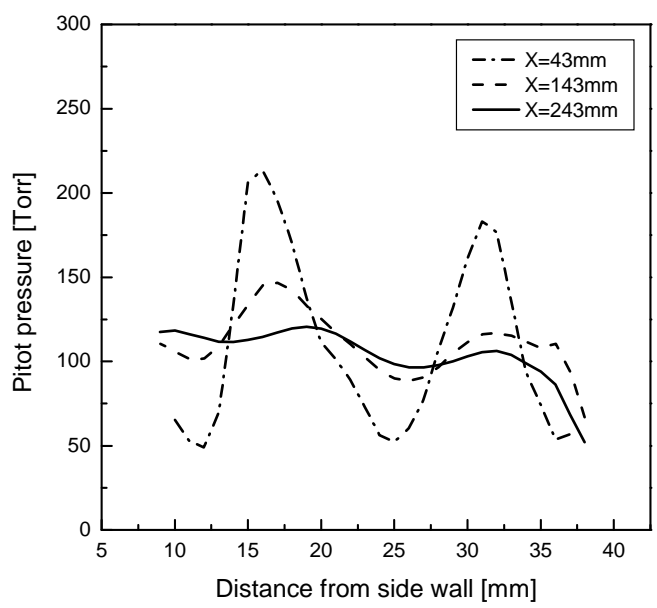

Fig. 2 Measured horizontal Pitot pressure profile for 5\% trip flow rate. Scanning was performed at three different positions from NEP (43 mm,143 mm, and $243 \mathrm{~mm}$ ).

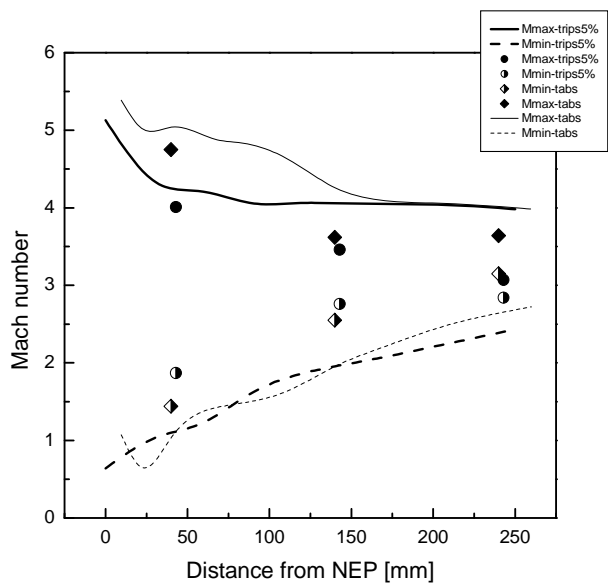

Fig. 3 Maximum and minimum Mach numbers as a function of distance from NEP. Dots indicate experimental results. Lines indicate CFD results.
Table 1 Summary of backpressure (Torr) test results for different trip flow rates and tab nozzles.

\begin{tabular}{|c|c|c|c|c|}
\hline & $3 \%$ & $5 \%$ & $7 \%$ & Tabs \\
\hline Start & 68.0 & 75.7 & 78.5 & 67.5 \\
\hline Unstart & 75.6 & 79.3 & 79.7 & 92.2 \\
\hline
\end{tabular}

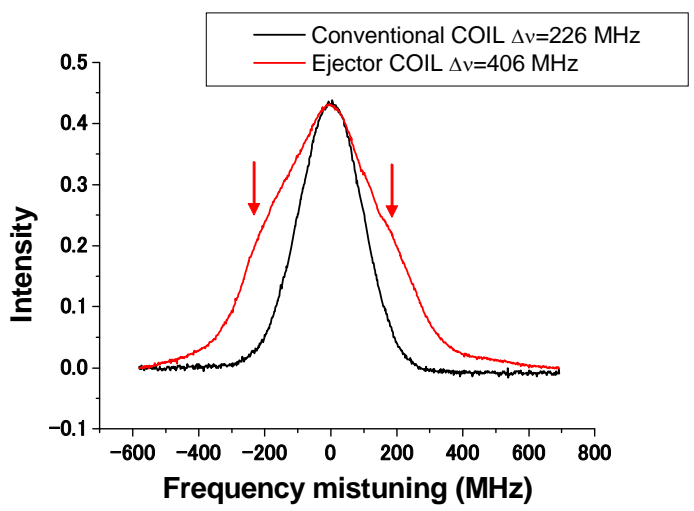

Fig. 4 Comparative study of gain spectra of ejector- and conventional-COIL.

was very small in the case of the trip-jets, while a difference of over 20 Torr was observed in the case of the tabs in our experiments.

\section{Hot-flow tests}

The gain measurements for different variants of nozzle bank and cavity were conducted on the several points along the flow direction. The measurements revealed that the maximum gain was obtained at the position around 100-150 mm from nozzle exit plane, and the gain cut-off length was more than $200 \mathrm{~mm}$. The maximum gain was around $0.6 \% / \mathrm{cm}$, and the gain spectrum width was about $400 \mathrm{MHz}$ which is much broader than the low-pressure conventional COIL. Figure 4 shows the observed gain spectrum. The gain spectrum is significantly distorted due to the presence of lateral flow components of the disturbed flow. The lateral flow components create the Doppler shifted gain line round about the central frequency component.

The lasing experiments were also conducted. The oxygen flow at the singlet oxygen generator (SOG) outlet was introduced to the laser cavity at Mach 1 and was subsequently accelerated by mixing with the $5: 1\left(\mathrm{~N}_{2}: \mathrm{O}_{2}\right)$ nitrogen diluent flow introduced to the cavity at Mach 5 through the nozzle bank of Fig. 1. The pressure of the SOG was approximately 20 Torr. In contrast with the conventional COIL, ${ }^{8,9}$ there was no diluent flow in the SOG. The yield of singlet oxygen was approximately $50 \%$ for the detachment yield of $70 \%{ }^{10)}$ The singlet oxygen yield is lower than that of the conventional COIL, ${ }^{8,9)}$ which was approximately $60 \%$. Lasing was achieved using a $4 \%$ out-coupling mirror $\left(\mathrm{M}_{1}\right)$ and a concave mirror $\left(\mathrm{M}_{2}\right)$ with a radius of curvature of $5 \mathrm{~m}$.The achieved laser 
power was about $3 \mathrm{~kW}$ at the titration $\left(\mathrm{I}_{2} / \mathrm{O}_{2}\right)$ of around $1.5-2 \%$. It is thought that the iodine condensation does not take places because of proper titration. The chemical efficiency of lasing was about $20 \%$.

\section{Conclusion}

A mixing system called the Uni-Lateral Trip-jet Induced Mixing Acceleration (ULTIMA) is presented herein in which trip-jets are proven to be quite effective for the mixing enhancement of the ejector-COIL. The mixing speed and the pressure recovery potential are comparable to the mixing tab scheme proposed by Yang et al. The advantages of the trips are that (i) a high-pressure iodine supply system is not necessary, (ii) the flow mixing speed can be controlled by the trip nitrogen flow rate and (iii) the stream is free from iodine condensation.

The ULTIMA system can produce Mach $3 \mathrm{O}_{2} / \mathrm{N}_{2}$ flow for which the total pressure is as high as 300 Torr. The observed Pitot pressure is 120 Torr.

The ULTIMA system can produce hot flow which reveals the gain of $0.6 \% / \mathrm{cm}$. The demonstrated laser power is $3 \mathrm{~kW}$.

The gain spectrum is significantly distorted due to the Doppler line splitting of lateral flow components of the disturbed flow. This effect should be considered in the analysis of flow temperature via the spectrum even in the conventional low-pressure COIL.

\section{References}

1) M. V. Zagidullin, V. D. Nikolaev, M. I. Svistun, N. A. Khvatov, G. D. Hager, and T. J. Madden: Quantum Electronics, 31 30, (2001).

2) V. D. Nikolaev, G. D. Hager, M.I. Svistun and M. V. Zagidullin: SPIE 5777 (2004) 160.

3) T. T. Yang, R. A. Dickerson, L. F. Moon, and Y. C. Hsia: AIAA paper 2425 (2000).

4) S. Hurlock: Proc. SPIE 4631 (2002) 101.

5) J. Marshall, K. Healey, B. Croker, K. Kendrick, T.T. Yang, Y.C. Hsia, R. A. Dickerson and L. Forman: SPIE 6101 (2006) 61011Z-1.

6) A. B. Witte, W. L. Shackleford, J. E. Broardwell, and J. E. Trost: AIAA Journal 13 (1975) 1408

7) L. E. Wilson: Proc. SPIE 76 (1976) 51.

8) K. Tei, D. Sugimoto, T. Ito, G. Watanabe, O. Vyskubenko, N. Takeuchi, and S. Muto: Proc. SPIE 5777 (2004) 281.

9) N. Takeuchi, D. Sugimoto, K. Tei, K. Nanri, and T. Fujioka: Jpn. J. Appl. Phys. 44 (2005) 895.

10) G. Watanabe, D. Sugimoto, K. Tei, and T. Fujioka: IEEE J. Quantum Electron. 40 (2004) 1030. 